\title{
Upregulation of ICAM-I by Plasmodium falciparum: in vitro and in vivo studies
}

\author{
W Graninger, J Prada, S Neifer, G Zotter, F Thalhammer, P G Kremsner
}

\begin{abstract}
Aims-To monitor the expression of intercellular adhesion molecule I (ICAMI) in vitro after stimulation of human macrophages with Plasmodium falciparum antigens, as well as the plasma concentrations of soluble ICAM-I (SICAM-I) in vivo in malarial patients. Methods-Human mononuclear leucocytes were cultured and stimulated for four hours with $300 \mathrm{ng} / \mathrm{ml}$ exogenous $P$ falciparum antigens. CD14 and CD54 (ICAM-I) expression was monitored using flow cytometry. Soluble ICAM-I (s ICAM-I) was also measured in the blood of 122 outpatients with malaria before and after treatment (Rio Branco, Acre, Brazil).
\end{abstract}

Results-ICAM-I expression increased from $15 \%$ to $375 \%$ after four hours of stimulation. When sICAM-I was analysed in the plasma of 122 patients with $P$ falciparum or Plasmodium vivax malaria by enzyme immunoassay, significant increases were found. These were more pronounced in patients with $P$ falciparum malaria, compared with healthy controls, and with the same patients four weeks after treatment.

Conclusion-ICAM-I expression may also be upregulated in human macrophages by exogenous Plasmodium antigens as well as by cytokines during the acute phase of malaria. sICAM-I concentrations are downregulated after treatment, probably caused by the absence of circulating Plasmodium antigens.

( Clin Pathol 1994;47:653-656)

Department of

Internal Medicine I,

Section of Infectious

Diseases, University

of Vienna, Austria

W Graninger

G Zotter

F Thalhammer

Landesinstitut für

Tropenmedizin

Berlin, Germany

J Prada

$S$ Neifer

P G Kremsner

Correspondence to:

Dr P G Kremsner,

Landesinstitut für

Tropenmedizin Berlin,

Engeldamm 62, 10179-

Engeldamm 62,
Berlin, Germany

Accepted for publication

25 November 1993
Intercellular adhesion molecule I (ICAM-I) is a sialylated glycoprotein with tissue specific differential glycosylation. It therefore has a wide molecular weight range between 70 and 120 kilodaltons. $^{1}$ ICAM-I can be readily induced in various cell types in response to cytokines such as $\gamma$-interferon (IFN- $\gamma$ ), tumour necrosis factor (TNF), and interleukin 1 (IL-1)..$^{2-4}$ The production of these last two can be upregulated by exogenous heat stable malarial antigens. ${ }^{56}$ ICAM-I also circulates in a soluble form (sICAM-I) in at least three different isoforms. ${ }^{1}$ Increased blood concentrations of sICAM-I have been observed in a range of different conditions, such as malignant melanoma, ${ }^{7}$ uveitis,${ }^{8}$ kidney and liver transplantation, ${ }^{910}$ acute myocardial infarction, ${ }^{11}$ rheumatoid arthritis, ${ }^{12}$ idiopathic lung fibrosis ${ }^{13}$ and neonatal sepsis. ${ }^{14}$ ICAM-I has also been identified as an endothelial cell surface receptor for erythrocytes infected with Plasmodium falciparum. ${ }^{15}$ While erythrocytes parasitised by Plasmodium vivax and immature ring-stage $P$ falciparum circulate without adhesion in the blood, erythrocytes with mature asexual stages of $P$ falciparum adhere to the endothelium. Soluble molecules of ICAM-I might interfere with the adherence of infected red blood cells, thus mitigating the course of $P$ falciparum malaria. ${ }^{16}$ By contrast, in $P$ vivax malaria, there is no such potential interference.

In this study the induction of ICAM-I expression in human macrophages was investigated after stimulation with malarial antigens, as well as the variations in the concentrations of sICAM-I in the blood of different groups of malarial patients before and after treatment.

\section{Methods}

For in vitro experiments, human mononuclear leucocytes were isolated from buffy coats from healthy adults, using Ficollhypaque density gradients, as described before. ${ }^{17}$ The separated cells were cultured and stimulated for four hours with malarial antigens. ${ }^{17}$ CD14 and CD54 (ICAM-I) expression was monitored by flow cytometry (FACSCAN, Becton Dickinson, Heidelberg, Germany).

One hundred and twenty two outpatients attending a health care centre in Rio Branco, Acre, Brazil had either untreated, uncomplicated $P$ falciparum malaria $(n=44$; nine women, 35 men; median age 22 years, range $16-50$ years) or $P$ vivax malaria $(n=78 ; 18$ females, 60 males; median age 22 years, range 3-81 years). In an arbitrary classification, ${ }^{18}$ patients with $P$ falciparum malaria were considered "non-immune" if they had had two or fewer malaria attacks during their life $(n=$ 28). Those who had had malaria three or more times in the preceding two years were classified as "semi-immune" $(n=16)$.

Fifteen healthy subjects from Rio Branco of similar gender and age distribution served as controls. None of them developed parasitaemia during the follow up period of 28 days.

All the patients infected with $P$ falciparum received oral clindamycin $5 \mathrm{mg} / \mathrm{kg}$ twice daily for five consecutive days, as described elsewhere. ${ }^{1920}$ Those infected with $P$ vivax received a total of $25 \mathrm{mg} / \mathrm{kg}$ oral chloroquine 
for two days and $0.25 \mathrm{mg} / \mathrm{kg}$ primaquine daily for 14 consecutive days. Plasma samples were collected from all patients on admission (day 0 ) and 28 days after treatment had started and stored at $-20^{\circ} \mathrm{C}$ until analysis.

Parasitaemia was determined by drawing $10 \mu \mathrm{l}$ blood (fingerprick), distributed equally on an $18 \times 10 \mathrm{~mm}$ area of a glass slide. After Giemsa staining, the parasite species was identified and the number of parasites counted per 100 oil-immersion fields ( $x$ 1000). The count was multiplied by four to yield the number of parasites per $1 \mu \mathrm{l}$ of blood. $^{21}$

Plasma concentrations of $\alpha_{1}$-proteinase inhibitor (API), $\alpha_{1}$ acid glycoprotein (AAG), and $C$-reactive protein (CRP) were measured by radioimmunodiffusion using commercially available test kits (Partigen, Behring Austria). Plasma concentrations of sICAM-I were assayed using a commercially available enzyme immunoassay (Bender, Austria). All samples were tested in duplicate and SICAM-I concentrations were determined by comparing the mean absorption of duplicate samples with that of a standard curve. The sensitivity of the test was about $0.5 \mathrm{ng} / \mathrm{ml}$.

Statistical analysis was performed using the Mann-Whitney U test and the Spearman rank correlation coefficient. The level of significance was set at $0 \cdot 05$.

\section{Results}

The effect of $P$ falciparum exogenous antigens on the in vitro expression of ICAM-I by human macrophages was analysed using fluorescein isothiocyanate (FITC) labelled anti-CD54 monoclonal antibodies in flow cytometry. The results of the experiment with maximal induction of ICAM-I are shown in fig 1. The separated human mononuclear leucocytes were incubated for four hours at $37^{\circ} \mathrm{C}$ with CRPMI medium without (fig 1A) and with (fig 1B) serum free preparations of $P$ fal- ciparum exogenous antigens at a standard concentration of $300 \mathrm{ng} / \mathrm{ml}$. Malarial antigens induced variable increases in ICAM-I expression $(15 \%$ to $375 \%)$. Most of the cells showing upregulated ICAM-I expression were monocytes or macrophages, determined using simultaneously phycoerythrin (PE) labelled anti-CD14 antibodies.

In the study of malarial patients in Acre, Brazil, all patients had been radically cured by day 28 of follow up. The concentrations of sICAM-I in plasma from the different groups of analysed patients before and after treatment are shown in fig 2 . Median values from "non-immune" (G2) and "semi-immune" (G3) patients infected with $P$ falciparum were compared with the corresponding median values from those infected with $P$ vivax (G4) and with healthy controls (G1). Significantly lower values were detected in the control group than in both the malarial groups. Both the "non-immunes" and the "semi-immunes" of the patients infected with $P$ falciparum had significantly higher concentrations of sICAMI than patients infected with $P$ vivax before and after treatment. There was no correlation between parasitaemia and SICAM-I concentrations in any of the groups of patients studied. sICAM concentrations were still increased 28 days after treatment in all the patients and were most pronounced in infections with $P$ falciparum.

The concentrations of acute phase reactants in plasma were analysed in all patients before and after treatment (table). Blood API concentrations were significantly lower in the control group than in "non-immune" $P$ falciparum and $P$ vivax malarial patients. There was no significant difference between the subgroups of patients infected with $P$ falciparum. A correlation between parasitaemia and API concentrations was detected only in those with $P$ falciparum malaria. Plasma AAG concentrations were significantly increased in all malarial patients. "Non-immune" patients

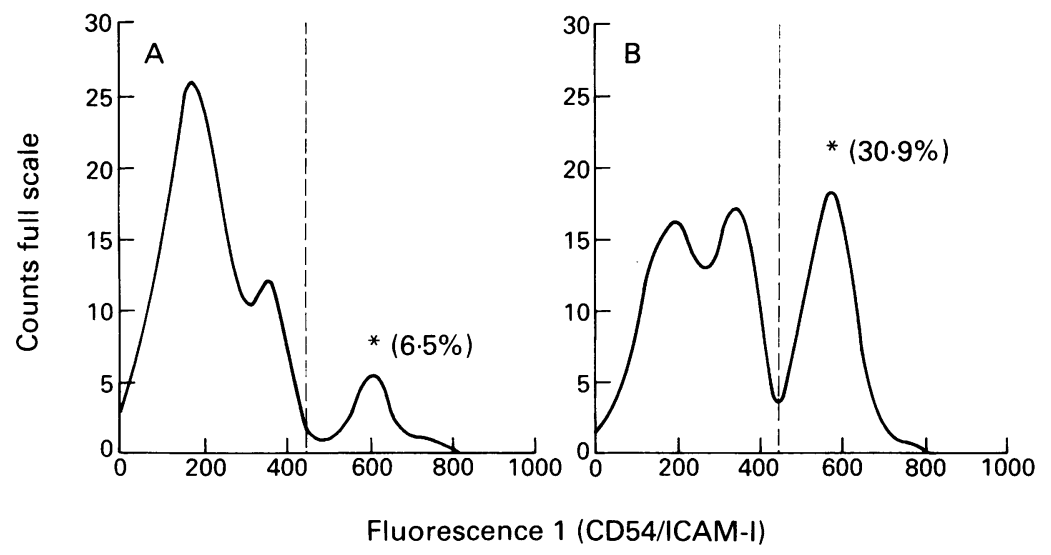

Figure 1 CD54 (ICAM-I) fluorescence patterns in human mononuclear leucocytes after four hours of incubation without $(A)$ and with $(B)$ exogenous antigens from $P$ falciparum at a concentration of $300 \mathrm{ng} / \mathrm{ml}$. The cell fractions indicated by * were mainly identified as monocytes or macrophages by using simultaneously phycoerythrin (PE) labelled antiCD14 monoclonal antibodies as fluorescence 2. The fluorescence patterns are the results of the experiment with maximal induction of ICAM-I, using FITC labelled anti-CD54 (ICAM-I) monoclonal antibodies as fluorescence 1.

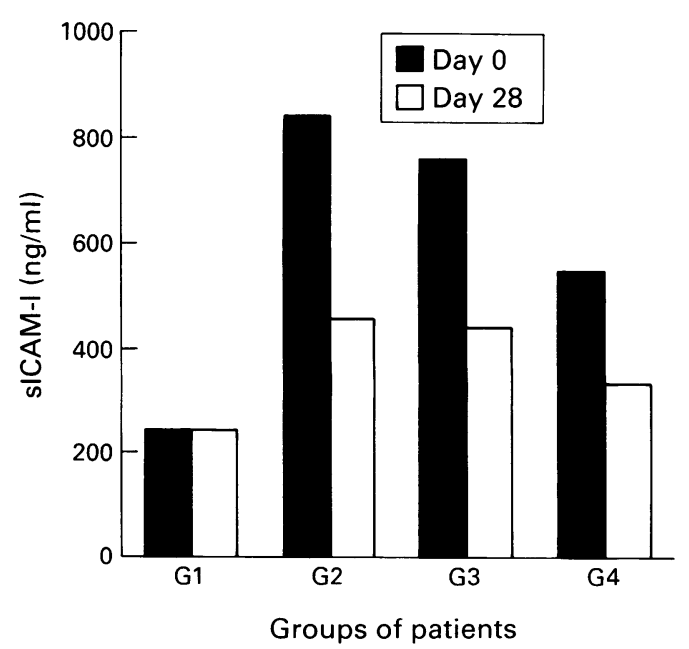

Figure 2 Soluble ICAM-I concentrations in plasma of patients infected with $P$ falciparum and $P$ vivax before (day 0) and after treatment (day 28). Values are medians for each group: $G 1$ = healthy controls, $G 2=$ "nonfor each group: $G 1=$ healthy controls, $G 2=$ "non-
immune" $P$ falciparium infected patients, $G 3=$ "semiimmune" $P$ falciparum infected patients, $G 4=P$ vivax malaria patients. 
Acute phase proteins from serum of patients infected with $P$ falciparum and $P$ vivax before (day 0) and after treatment (day 28)

\begin{tabular}{|c|c|c|c|c|}
\hline & \multirow[b]{2}{*}{$\begin{array}{l}\text { Controls } \\
(n=15)\end{array}$} & \multicolumn{2}{|l|}{$P$ falciparum } & \multirow[b]{2}{*}{$\begin{array}{l}P \text { vivax } \\
(n=78)\end{array}$} \\
\hline & & $\begin{array}{l}\text { "Semi-immunes" } \\
(n=16)\end{array}$ & $\begin{array}{l}\text { "Non-immunes" } \\
(n=28)\end{array}$ & \\
\hline Parasitaemia-(0) & 0 & $\begin{array}{l}1710 \\
(12-10764)\end{array}$ & $\begin{array}{l}3112 \\
(16-32680)\end{array}$ & $\begin{array}{l}3000 \\
(10-18000)\end{array}$ \\
\hline API-(0) & $\begin{array}{l}209 \\
(161-271)\end{array}$ & $\begin{array}{l}252 \\
(135-236)\end{array}$ & $\begin{array}{l}297^{\star}+t \\
(133-411)\end{array}$ & $\begin{array}{l}285^{\star}+t \\
(167-502)\end{array}$ \\
\hline API-(28) & $\begin{array}{l}184 \\
(129-255)\end{array}$ & 211 & $\begin{array}{l}226^{\star} \\
(126-300)\end{array}$ & $\begin{array}{l}235^{\star} \\
(135-338)\end{array}$ \\
\hline AAG-(0) & $\begin{array}{l}72 \\
(38-148)\end{array}$ & $\begin{array}{l}120^{\star}+t \\
(41-175)\end{array}$ & $\begin{array}{l}146^{\star}+\dagger \\
(45-269)\end{array}$ & $\begin{array}{l}122^{\star}+\dagger \\
(46-208)\end{array}$ \\
\hline AAG-(28) & $\begin{array}{l}71 \\
(44-121)\end{array}$ & $\begin{array}{l}61+\ddagger \\
(23-94)\end{array}$ & $\begin{array}{l}78 \\
(30-640)\end{array}$ & $\begin{array}{l}85 \\
(27-272)\end{array}$ \\
\hline CRP-(0) & $\begin{array}{l}0 \\
(0-3 \cdot 0)\end{array}$ & $\begin{array}{l}2 \cdot 3^{\star}+\neq+t^{\star \star} \\
(0 \cdot 6-5 \cdot 1)\end{array}$ & $\begin{array}{l}5 \cdot 4^{\star}+t^{\star \star} \\
(0-9 \cdot 0)\end{array}$ & $\begin{array}{l}5 \cdot 2^{\star}+t^{\star \star} \\
(0-12 \cdot 2)\end{array}$ \\
\hline CRP-(28) & $(0-0 \cdot 7)$ & $\begin{array}{l}0 \\
(0-0.4)\end{array}$ & $\begin{array}{l}0 \\
(0-7 \cdot 8)\end{array}$ & $\begin{array}{l}0 \\
(0-3 \cdot 2)\end{array}$ \\
\hline
\end{tabular}

Values are expressed as median and range. Parasitaemia is expressed in parasites per $\mu \mathrm{l}$ blood API, AAG, and CRP in $\mathrm{mg} / 100 \mathrm{ml}$ plasma.

¿Difference from controls was significant $(p<0.05)$

†Difference from "non-immunes" was significant.

Difference from $P$ vivax group was sionificant.

+ Difference from day 28 value was significant.

$\star \star$ Correlation with parasitaemia was significant.

with $P$ falciparum had higher initial concentrations than "semi-immune" patients, or than patients infected with $P$ vivax. There was a positive correlation between parasitaemia and AAG plasma concentrations in patients with $P$ falciparum. CRP concentrations discriminated best between the control group and all subgroups of malarial patients. There were also significant differences between "semiimmune" and "non-immune" patients infected with $P$ falciparum, and between the "semi-immune" subgroup and the $P$ vivax malarial patients. Correlations between the CRP concentrations and parasitaemia were found in both subgroups of $P$ falciparum patients, as well as in the $P$ vivax patients.

\section{Discussion}

As far as we are aware, this is the first report about the induction of ICAM-I by malarial antigens. This effect is to be expected, however, as exogenous malarial antigens have been found to induce both TNF and IL-1 secretion, ${ }^{56}$ which would consequently induce the expression of ICAM-I. Remarkably, the maximal increases in ICAM-I expression found after four hours of stimulation with malarial antigens correspond with maximal TNF production as a response to in vitro stimulation of human mononuclear leucocytes with $P$ falciparum exogenous antigens under similar conditions. ${ }^{17}$ Therefore, it is not possible to conclude whether ICAM-I upregulation is caused by the antigens directly or indirectly via an autocrine stimulatory pathway involving TNF and IL-1.

The discovery of ICAM-I as one of the adhesion molecules responsible for sequestration in $P$ falciparum malaria, ${ }^{22}$ as well as our own in vitro results, led to a further investigation of sICAM-I plasma concentrations in malarial patients. Indeed, high sICAM-I concentrations were found in patients with $P$ falciparum malaria, and these decreased gradually after treatment with clindamycin. Although to a lesser degree, sICAM-I concentrations were also increased in patients with $P$ vivax malaria. In the latter, no parasite sequestration occurs, raising the question of how sICAM-I concentrations and cellular expression of ICAM-I are associated with each other. As to the numerous conditions in which increased sICAM-I concentrations have been described, it is obvious that sICAM-I plasma concentrations are related to the amounts of cytokines released. The plasma concentrations of the acute phase proteins API, AAG, and CRP, induced by monokines, behave in a similar manner to sICAM-I, with the highest concentrations seen in "non-immune" patients with $P$ falciparum malaria and the lowest concentrations in the "semi-immune" $P$ falciparum and $P$ vivax groups. However, unlike sICAM-I, differences between the increased concentrations of acute phase proteins were not significant in patients with $P$ falciparum or $P$ vivax malaria. This indicates that there might be specific differences associated with the role of SICAM-I in both groups of malarial patients.

The high concentrations of API, AAG, and CRP returned to lower levels comparable with those of healthy controls within four weeks of starting treatment, and this was especially noticeable for CRP, where a correlation with parasitaemia was also observed. This agrees with other studies, which have also shown a link between parasitaemia and CRP concentrations in patients infected with $P$ falciparum. ${ }^{23-25}$ Plasma sICAM-I concentrations behaved in a similar manner to those of acute phase proteins, but no correlation was found between sICAM-I concentrations and parasitaemia or the concentrations of any of the acute phase proteins analysed. This might have been due to the different kinetics of production and metabolism. Moreover, the upregulation of sICAM-I expression during the acute phase of malaria can be attributed to an increased release of cytokines, ${ }^{26}$ but whether sICAM-I has any any pathogenic role in malaria or merely represents an unspecific response to enhanced cytokine secretion and the presence of malaria antigens remains to be determined.

We are grateful to B Jacob and F Daniels for their technical assistance, to $L$ Lehman and $W$ Metzger for help and comments, and to $\mathrm{H} \mathrm{G}$ Heidrich for providing serum free $P$ falciparum exogenous antigens. We also thank the Krankenhaus Moabit, Berlin, for the kind donation of buffy coats for the preparation of human mononuclear leucocytes.

1 Rothlein R, Mainolfi EA, Czajkowski M, Marlin SD. A form of circulating ICAM-I in human serum. $\mathcal{F}$ Immuno 1991;147:3788-93.

2 Pigott R, Dillon LP, Hemingway IH, Gearing $\mathrm{AJH}$. Soluble E-selectin, ICAM-I and VCAM-I are present in the supernatants of cytokine activated cultured endothelial cells. Biochem Biophys Res Commun 1992;187:584-9.

3 Pober JS, Gimbrone MA, Lapierre LA, et al. Overlapping patterns for activation of human endothelial cells by interleukin 1, tumour necrosis factor and immune interinterleukin 1, tumour necrosis facto

4 Dustin ML, Rothlein R, Bhan AK, Dinarello CA, Springer TA. Induction by IL-1 and interferon-gamma: tissue distribution, biochemistry and function of a natural adherence molecule (ICAM-I). $f$ Immunol 1986;137:245-54

5 Taverne J, Bate CAW, Kwiatkowski D, Jakobsen PH, Playfair JHL. Two soluble antigens of Plasmodium falciparum induce tumor necrosis factor release from macrophages. Infect Immun 1990;58:2923-8.

6 Schofield L, Hackett F. Signal transduction in host cells by 
a glycosylphosphatidylinositol toxin of malaria parasites. $f$ Exp Med 1993;1:145-53.

7 Harning R, Mainolfi E, Bystryn JC, Henn M, Merluzzi VJ, Rothlein R. Serum levels of circulating intercellular adhesion molecule 1 in human malignant melanoma. Cancer Res 1991;51:5003-5.

8 Arocker-Mettinger E, Steurer-Georgiew L, Steuer M, et al. Circulating ICAM-I levels in serum of uveitis patients. Current Eye Res 1992;11:161-6.

9 Stockenhuber F, Gnant M, Götzinger P, et al. Soluble ICAM-I in renal and liver transplant recipients. Wien Med Wochenschr 1992;4:6.

10 Adams DH, Mainolf E, Elias E, Neuberger JM, Rothlein R. Detection of circulating intercellular adhesion molecule 1 after liver transplantation; evidence of local release within the liver during graft rejection. Transplantation 1993;55:83-7.

11 Gottsauner-Wolf $M$, Sochor H, Probst P, Röder S, Balcke $P$, Stockenhuber F. Preliminary data of sICAM-I in patients with acute myocardial infarction. Wien Med Wochenschr 1992;4:7.

12 Machold KP, Kiener HP, Graninger W, Graninger WB Soluble intercellular adhesion molecule 1 (sICAM-I) in patients with rheumatoid arthritis and systemic lupus erythematosus. Clin Immunol Immunopathol 1993;68. 74-8.

13 Shijubo N, Imai K, Aoki S, et al. Circulating intercellular adhesion molecule 1 (ICAM-I) antigen in sera of patients with idiopathic pulmonary fibrosis. Clin Exp Immunol 1992;89:58-62.

14 Kuster H, Degitz K. Circulating ICAM-I in neonatal sepsis Lancet 1993;341:506.

15 Berendt AR, Simmons DL, Tansey J, Newbold CI, Marsch K. Intercellular adhesion molecule 1 is an endothelial cell adhesion receptor for Plasmodium falciparum. Nature 1989;341:57-9.

16 Staunton DE, Ockenhouse CF, Sprinter TA. Soluble intercellular adhesion molecule 1-immunoglobulin G1 immunoadhesin mediates phagocytosis of malariaimmunoadhesin mediates phagocytosis of mal
infected erythrocytes. $\Im \operatorname{Exp}$ Med 1992;176:1471-6.
17 Prada J, Prager C, Neifer S, Bienzle U, Kremsner PG. Production of interleukin 6 by human and murin mononuclear leukocytes stimulated with Plasmodium antigens is enhanced by pentoxifylline and tumor necrosis factor secretion is reduced. Infect Immun 1993;61:2737-40.

18 Kremsner PG, Zotter GM, Feldmeier H, et al. Immune response in patients during and after Plasmodium falciparum infection. $\mathcal{F}$ Infect Dis 1990;161:1025-8.

19 Kremsner PG, Zotter GM, Feldmeier H, Graninger W Westerman RI, Rocha RM. Clindamycin treatment of Plasmodium falciparum malaria in Brazil. $\Im$ Antimicrob Plasmodium falciparum mather 1989;23:275-81.

20 Kremsner PG, Graninger W. Clindamycin in the treatment of experimental and human malaria. Rev Contemp Pharmacother 1992;3:275-9.

21 Kremsner PG, Zotter GM, Feldmeier H, Graninger W, Rocha RM, Wiedermann G. A comparative trial of three regimens for treating uncomplicated Plasmodium falciparum malaria in Acre, Brazil f Infect Dis 1988, 158:1368-71.

22 Ockenhouse CF, Ho $M$, Tandon NN, et al. Molecular basis of sequestration in severe and uncomplicated Plasmodium falciparum malaria: Differential adhesion of infected erythrocytes to CD 36 and ICAM-I. 7 Infect Dis 1991;164:163-9.

23 Graninger W, Thalhammer F, Hollenstein U, Zotter GM, Kremsner PG. Serum protein concentrations in Plasmodium falciparum malaria. Acta Trop 1992 52:121-8.

24 Naik P, Voller A. Serum C-reactive protein levels and Plasmodium falciparum malaria. Trans Roy Soc Trop Med Hyg 1984;78:812-3.

25 Gillespie SH, Dow C, Raynes JG, Behrens RH, Chiodin PL, McAdam KPWJ. Measurement of acute phase proteins for assessing severity of Plasmodium falciparum teins for assessing severity of Plasmodia.

26 Harpaz R, Edelman R, Wasserman SS, Levine MM, Davi JR, Sztein MB. Serum cytokine profiles in experimental human malaria. F Clin Invest 1992;90:515-23. 